\title{
Effective-Resistance Preserving Spectral Reduction of Graphs
}

\author{
Zhiqiang Zhao \\ Michigan Technological University \\ Houghton, Michigan \\ qzzhao@mtu.edu
}

\author{
Zhuo Feng \\ Michigan Technological University \\ Houghton, Michigan \\ zhuofeng@mtu.edu
}

\begin{abstract}
This paper proposes a scalable algorithmic framework for effectiveresistance preserving spectral reduction of large undirected graphs. The proposed method allows computing much smaller graphs while preserving the key spectral (structural) properties of the original graph. Our framework is built upon the following three key components: a spectrum-preserving node aggregation and reduction scheme, a spectral graph sparsification framework with iterative edge weight scaling, as well as effective-resistance preserving postscaling and iterative solution refinement schemes. By leveraging recent similarity-aware spectral sparsification method and graphtheoretic algebraic multigrid (AMG) Laplacian solver, a novel constrained stochastic gradient descent (SGD) optimization approach has been proposed for achieving truly scalable performance (nearlylinear complexity) for spectral graph reduction. We show that the resultant spectrally-reduced graphs can robustly preserve the first few nontrivial eigenvalues and eigenvectors of the original graph Laplacian and thus allow for developing highly-scalable spectral graph partitioning and circuit simulation algorithms.
\end{abstract}

\section{KEYWORDS}

Spectral graph theory, graph reduction, spectral partitioning

\section{ACM Reference Format:}

Zhiqiang Zhao and Zhuo Feng. 2019. Effective-Resistance Preserving Spectral Reduction of Graphs. In The 56th Annual Design Automation Conference 2019 (DAC '19), fune 2-6, 2019, Las Vegas, NV, USA. ACM, New York, NY, USA, 6 pages. https://doi.org/10.1145/3316781.3317809

\section{INTRODUCTION}

Recent research shows that by leveraging the key spectral properties of eigenvalues and eigenvectors of graph Laplacians, more efficient algorithms can be developed for tackling many graphrelated computing tasks [21]. For example, spectral methods can potentially lead to much faster algorithms for solving sparse matrices [18, 24], numerical optimization [6], graph analytics [13], machine learning [8], as well as very-large-scale integration (VLSI) computer-aided design (CAD) $[11,22]$. To this end, spectral sparsification of graphs has been extensively studied in the past decade $[3,19]$ to allow computing almost-linear-sized ${ }^{1}$ subgraphs or sparsifiers that can robustly preserve the spectrum, such as the eigenvalues and eigenvectors of the original Laplacian. The sparsified graphs retain the same set of vertices but much fewer edges, which

\footnotetext{
${ }^{1}$ The number of vertices (nodes) is similar to the number of edges.

Permission to make digital or hard copies of all or part of this work for personal or classroom use is granted without fee provided that copies are not made or distributed for profit or commercial advantage and that copies bear this notice and the full citation on the first page. Copyrights for components of this work owned by others than ACM must be honored. Abstracting with credit is permitted. To copy otherwise, or republish to post on servers or to redistribute to lists, requires prior specific permission and/or a fee. Request permissions from permissions@acm.org.

DAC '19, June 2-6, 2019, Las Vegas, NV, USA

(C) 2019 Association for Computing Machinery.

ACM ISBN 978-1-4503-6725-7/19/06 ...\$15.00

https://doi.org/10.1145/3316781.3317809
}

can be regarded as ultra-sparse graph proxies and have been leveraged for developing a series of nearly-linear-time numerical and graph algorithms $[6,18,19]$.

In this paper, we introduce a scalable algorithmic framework for effective-resistance preserving spectral reduction of graphs, to allow dramatically reducing the size (both nodes and edges) of undirected graphs, while preserving the key spectral (structural) properties of the original graph, such as the Laplacian eigenvalues/eigenvectors, the cuts in the graph, effective resistances between nodes, etc. The spectrally-reduced graphs will immediately lead to the development of much faster numerical and graph-related algorithms. For example, spectrally-reduced social (data) networks may allow for more efficiently modeling, mining and analysis of large social (data) networks, spectrally-reduced neural networks allow for more scalable model training and processing in emerging machine learning tasks, spectrally-reduced circuit networks may lead to more efficient simulation, optimization and verification of large integrated circuit (IC) systems, etc.

Our approach consists of three key phases: 1) a highly-scalable spectrum-preserving node aggregation and reduction phase, 2) a spectral graph sparsification phase with iterative edge weight scaling, and 3) an effective-resistance preserving post-scaling phase. To achieve truly scalable (nearly-linear time) performance for spectral graph reduction, we leverage recent similarity-aware spectral graph sparsification method [11], graph-theoretic algebraic multigrid (AMG) Laplacian solver [15, 24], a novel constrained stochastic gradient descent (SGD) optimization approach, and an effectiveresistance preserving post-scaling scheme. The major contribution of this work has been summarized as follows:

(1) To well preserve the key spectral properties of the original graph in the reduced graph, a nearly-linear time spectrum-preserving node aggregation and reduction scheme is proposed for robustly constructing reduced graphs based on algebraic distance metric [5]. (2) A scalable framework for spectral graph sparsification and iterative edge weight scaling is introduced for assuring sparsity as well as preserving the original spectral properties in the reduced graphs by leveraging a novel constrained SGD optimization approach and a recent similarity-aware spectral sparsification method [11].

(3) We introduce a simple yet effective procedure for refining solutions computed by using spectrally-reduced graphs, which immediately allows using much smaller graphs in many numerical and graph algorithms while achieving superior solution qualities.

The rest of this paper is organized as follows: Section 2 provides a brief introduction to spectral graph sparsification; Section 3 presents the proposed effective-resistance preserving spectral graph reduction approach and its complexity analysis. Extensive experimental results have been demonstrated in Section 4, which is followed by the conclusion of this work in Section 5 .

\section{PRELIMINARIES}

Spectral graph sparsification aims to find a spectrally-similar subgraph (sparsifier) $P=\left(V, E_{P}, w_{P}\right)$ that has the same set of vertices of the original graph $G=\left(V, E_{G}, w_{G}\right)$, but much fewer edges. We 


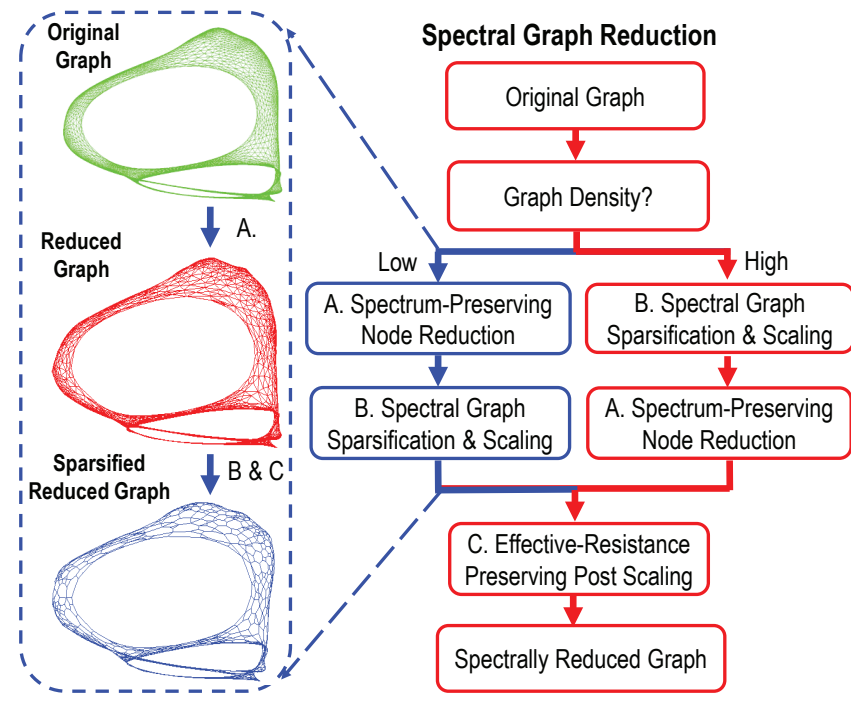

Figure 1: Overview of the proposed effective-resistance preserving spectral graph reduction method.

say $G$ and its subgraph $P$ are $\sigma$-spectrally similar if the following condition holds for all real vectors $\mathbf{x} \in \mathbb{R}^{V}$

$$
\frac{\mathbf{x}^{\top} \mathbf{L}_{P} \mathbf{x}}{\sigma} \leq \mathbf{x}^{\top} \mathbf{L}_{G} \mathbf{x} \leq \sigma \mathbf{x}^{\top} \mathbf{L}_{P} \mathbf{x}
$$

where $\mathbf{L}_{G}$ and $\mathbf{L}_{P}$ denote the symmetric diagonally dominant (SDD) Laplacian matrices of graph $G$ and $P$, respectively. Define the relative condition number as $\kappa\left(\mathbf{L}_{G}, \mathbf{L}_{P}\right)=\lambda_{\max } / \lambda_{\min }$, where $\lambda_{\max }$ and $\lambda_{\min }$ are the largest and smallest nonzero eigenvalues of

$$
\mathbf{L}_{G} \mathbf{u}=\lambda \mathbf{L}_{P} \mathbf{u},
$$

where $\mathbf{u}$ is the generalized eigenvector of $\lambda$. It can be further shown that $\kappa\left(\mathbf{L}_{G}, \mathbf{L}_{P}\right) \leq \sigma^{2}$, which indicates that a smaller relative condition number or $\sigma^{2}$ corresponds to a higher spectral similarity. Recent nearly-linear time spectral sparsification algorithm leverages spectral perturbation analysis to construct nearly-linear-sized spectrally-similar subgraphs $[10,11]$, which leads to the development of much faster SDD matrix solvers [24] and spectral graph partitioning algorithms [11]. However, whether the spectrally-similar subgraphs can be further simplified or improved by taking advantage of the ultra-sparse subgraph topology as well as edge weight scaling still remains an open question.

\section{EFFECTIVE-RESISTANCE PRESERVING SPECTRAL GRAPH REDUCTION}

\subsection{Overview of Our Approach}

As illustrated in Figure 1, the proposed spectral graph reduction approach consists the following three phases: Phase (A) will determine the fine-to-coarse graph mapping operator based on spectral node similarity in the (undirected or directed) graph; Phase (B) will extract spectral sparsifiers of the original or reduced graph and iteratively scale up edge weights in the sparsified (reduced) graph to better match the key graph spectral (structural) properties, such as the eigenvectors of graph Laplacians; Phase (C) will globally scale up the sparsified (reduced) graph for matching the original Laplacian eigenvalues and effective resistances between nodes. Since
Table 1: Symbols used in the paper

\begin{tabular}{|c|c|c|c|}
\hline \multicolumn{4}{|c|}{ Symbol Table } \\
\hline Symbol & Denotation & Symbol & Denotation \\
\hline$G=\left(V, E_{G}, w_{G}\right)$ & The Orig. Input Graph & $\mathrm{L}_{G}$ & Laplacian of $G$ \\
$P=\left(V, E_{P}, w_{P}\right)$ & Spectrally-Spar. $G$ & $\mathrm{~L}_{P}$ & Laplacian of $P$ \\
$R=\left(V_{R}, E_{R}, w_{R}\right)$ & Reduced $G$ w/o spar. & $\mathrm{L}_{R}$ & Laplacian of $R$ \\
$S=\left(V_{R}, E_{S}, w_{S}\right)$ & Reduced $G$ w/ spar. & $\mathrm{L}_{S}$ & Laplacian of $S$ \\
$\mathrm{H}_{G}^{R} \in \mathbb{R}_{R} \times V$ & G-to-R mapping matrix & $\mathrm{H}_{R}^{G} \in \mathbb{R}^{V \times V_{R}}$ & R-to-G mapping matrix \\
\hline
\end{tabular}

the spectral node similarity metric based on algebraic distance cannot be reliably applied to dense graphs [5], our approach will first examine the average and maximum node degrees in the original graph: if the original graph is relatively sparse, Phases (A) to (C) will be performed in sequence as shown in Figure 1; otherwise, if the original graph is too dense, Phase (B) for spectral graph sparsification and edge scaling will be performed first, which is followed by Phase (A) and Phase (C).

In the following, we assume that $G=\left(V, E_{G}, w_{G}\right)$ is a weighted, undirected, and connected graph, $P=\left(V, E_{P}, w_{P}\right)$ is the spectrally sparsified graph of $G, R=\left(V_{R}, E_{R}, w_{R}\right)$ is the reduced graph of $G$ without sparsification, and $S=\left(V_{R}, E_{S}, w_{S}\right)$ is the final reduced graph of $G$. The Laplacian matrices of the corresponding graphs have been shown in Table 1 that also includes the fine-to-coarse (G-to-R) graph mapping matrix denoted by $\mathbf{H}_{G}^{R}$ as well as the coarseto-fine (R-to-G) graph mapping matrix denoted by $\mathbf{H}_{R}^{G}$.

\subsection{Phase (A): Spectrum-Preserving Node Reduction}

Spectral node affinity metric. To generate the reduced graph based on the original graph, a spectrum-preserving node aggregation scheme is applied based on spectral node affinity $a_{p, q}$ defined as follows for neighboring nodes $p$ and $q[5,15]$ :

$$
a_{p, q}=\frac{\left\|\left(\mathbf{X}_{\mathbf{p}}, \mathbf{X}_{\mathbf{q}}\right)\right\|^{2}}{\left(\mathbf{X}_{\mathbf{p}}, \mathbf{X}_{\mathbf{p}}\right)\left(\mathbf{X}_{\mathbf{q}}, \mathbf{X}_{\mathbf{q}}\right)},\left(\mathbf{X}_{\mathbf{p}}, \mathbf{X}_{\mathbf{q}}\right)=\Sigma_{\mathbf{k}=1}^{\mathrm{K}}\left(\mathbf{x}_{\mathbf{p}}^{(\mathbf{k})} \cdot \mathbf{x}_{\mathbf{q}}^{(\mathbf{k})}\right),
$$

where $\mathbf{X}=\left(\mathbf{x}^{(\mathbf{1})}, \ldots, \mathbf{x}^{(\mathbf{K})}\right)$ includes $K$ test vectors computed by applying a few Gauss-Seidel (GS) relaxations for solving the linear system of equations $\mathrm{L}_{\mathrm{G}} \mathbf{x}^{(\mathrm{i})}=0$ for $i=1, \ldots, K$ with $K$ random vectors that are orthogonal to the all-one vector 1 or equivalently satisfying $\mathbf{1}^{\top} \mathbf{x}^{(\mathbf{i})}=\mathbf{0}$. Let $\tilde{\mathbf{x}}^{(\mathbf{i})}$ denote the approximation of the true solution $\mathbf{x}^{(\mathbf{i})}$ after applying several GS relaxations to $\mathbf{L}_{G} \mathbf{x}^{(\mathbf{i})}=0$. Due to the smoothing property of GS relaxation, the latest error can be expressed as $\mathbf{e}_{\mathrm{s}}^{(\mathbf{i})}=\mathbf{x}^{(\mathbf{i})}-\tilde{\mathbf{x}}^{(\mathbf{i})}$, which will only contain the smooth (low-frequency) modes of the initial error, while the oscillatory (high-frequency) modes of the initial error will be effectively eliminated [4]. Based on the $K$ smoothed vectors in $\mathrm{X}$, it is possible to embed each node into a $K$-dimensional space such that node $p$ and node $q$ are considered spectrally similar to each other if their low-dimensional embedding vectors $\mathbf{x}_{\mathbf{p}} \in \mathbb{R}^{K}$ and $\mathbf{x}_{\mathbf{q}} \in \mathbb{R}^{K}$ are highly correlated. Spectrally-similar nodes $p$ and $q$ can be then aggregated together for node reduction purpose.

Spectral similarity between nodes. It has been shown that the node affinity metric $a_{p, q}$ can usually effectively reflect the distance or strength of connection between nodes $p$ and $q$ in a graph [15]: a larger $a_{p, q}$ value indicates a higher spectral similarity (correlation) between nodes $p$ and $q$. The above affinity metric can be better understood by looking at the algebraic distance $d_{p, q}$ between node $p$ and $q$ computed by $d_{p, q}=1-a_{p, q}$ that can be used to represent the geometric distance in grid-structured graphs. For example, consider an $n$-node $1 \mathrm{D}$ path graph with a discretization 
size of $h=1 / n$. It can be shown that $d_{p, q}$ is proportional to $(p-q)^{2} *$ $h^{2}$ in the discretized Poisson equation in which nodes locate at $t * h$, for $t=0,1 \ldots, n$ and $h \Rightarrow 0$. Consequently, the nodes with large affinities or small algebraic distances should be aggregated together to form the nodes in the reduced graph. Once the node aggregation schemes are determined, the graph mapping operators $\left(\mathbf{H}_{\mathrm{G}}^{\mathrm{R}}\right.$ and $\left.\mathbf{H}_{\mathbf{R}}^{\mathrm{G}}\right)$ can be obtained and leveraged for constructing spectrally-reduced graphs. For example, the reduced Laplacian can be computed by $\mathbf{L}_{\mathrm{R}}=\mathrm{H}_{\mathrm{G}}^{\mathrm{R}} \mathrm{L}_{\mathrm{G}} \mathrm{H}_{\mathrm{R}}^{\mathrm{G}}$, which uniquely defines the reduced graph. We emphasize that the node aggregation (reduction) scheme based on the above spectral node affinity calculations will have a (linear) complexity of $O\left(\left|E_{G}\right|\right)$ and thus allow preserving the spectral (global or structural) properties of the original graph in the reduced graph in a highly efficient and effective way: the smooth components in the first few Laplacian eigenvectors can be well preserved after node aggregation, which is key to preserving the original graph spectra in the reduced graphs.

Spectral node similarity for dense graphs. The above node reduction scheme based on the algebraic distance metric may not be reliable when applied to dense graph problems. Since each node in the dense graph will typically connect to many other nodes, running a few GS relaxations will result in many nodes seemingly close to each other and can lead to rather poor node aggregation results. For example, an extreme case is to directly apply the above node aggregation scheme to a complete graph where each node has $|V|-1$ edges connecting to the rest of the nodes: since applying GS relaxations will immediately assign similar values to all nodes, no meaningful clusters of nodes can be identified. As shown in our experiment results, it is not possible to use the above node affinity metric for aggregating nodes in the "appu" graph [7] that has high average node degrees $\left(\left|E_{G}\right| /|V| \approx 70\right)$. To this end, we propose to perform a spectral sparsification and scaling procedure before applying the spectral node aggregation (reduction) phase. Such a scheme will first extract ultra-sparse yet spectrally-similar subgraphs and subsequently aggregate nodes into clusters using the above node affinity metric. As a result, the spectral graph reduction flow proposed in this work can be reliably applied to both sparse and dense graphs, as shown in Figure 1.

\subsection{Phase (B): Spectral Graph Sparsification and Scaling}

In the following, we consider the case for spectral reduction of dense graphs: the spectral topology sparsification and subgraph scaling procedure in Phase (B) will be performed before applying the spectral node reduction scheme in Phase (A).

3.3.1 Spectral Graph Topology Sparsification. Recent research shows that every graph has a $\sigma$-similar spectral graph sparsifier that can be constructed by recovering $O\left(\frac{m \log n \log \log n}{\sigma^{2}}\right)$ off-tree edges to a low-stretch spanning tree (LSST) [9] in nearly-linear time using a spectral perturbation framework $[10,11]$, where $m=\left|E_{G}\right|$ and $n=|V|$. To identify the key off-tree edges to be added to the LSST for dramatically reducing spectral distortion (the total stretch), a spectral embedding scheme using approximate generalized eigenvectors has been introduced in [10], which is based on the following spectral perturbation analysis:

$$
\mathbf{L}_{G}\left(\mathbf{u}_{i}+\delta \mathbf{u}_{i}\right)=\left(\lambda_{i}+\delta \lambda_{i}\right)\left(\mathbf{L}_{P}+\delta \mathbf{L}_{P}\right)\left(\mathbf{u}_{i}+\delta \mathbf{u}_{i}\right),
$$

where a perturbation $\delta \mathbf{L}_{P}$ is applied to $\mathrm{L}_{P}$, which results in perturbations in generalized eigenvalues $\lambda_{i}+\delta \lambda_{i}$ and eigenvectors $\mathbf{u}_{i}+\delta \mathbf{u}_{i}$ for $i=1, \ldots, n$, respectively. We assume that the descending eigenvalues of $\mathrm{L}_{\mathrm{P}}^{+} \mathrm{L}_{\mathrm{G}}$ are $\lambda_{\max }=\lambda_{1} \geq \lambda_{2} \geq \cdots \geq \lambda_{n}=\lambda_{\text {min }}$, then the first-order perturbation analysis [10] leads to

$$
-\frac{\delta \lambda_{i}}{\lambda_{i}}=\mathbf{u}_{i}^{\top} \delta \mathbf{L}_{P} \mathbf{u}_{i},
$$

which indicates that the reduction of $\lambda_{i}$ is proportional to the Laplacian quadratic form of $\delta \mathbf{L}_{P}$ with the generalized eigenvector $\mathbf{u}_{i}$.

3.3.2 Bounded Mismatch in the Subgraph. We note that (5) can be understood with the following Courant-Fischer theorem for generalized eigenvalue problems:

$$
\lambda_{1}=\max _{\substack{|\mathbf{x}| \neq 0 \\ \mathbf{x}^{\top} \mathbf{1}=0}} \frac{\mathbf{x}^{\top} \mathbf{L}_{G} \mathbf{x}}{\mathbf{x}^{\top} \mathbf{L}_{P} \mathbf{x}} \geq \max _{\substack{|\mathbf{x}| \neq 0 \\ x(p) \in\{0,1\}}} \frac{\mathbf{x}^{\top} \mathbf{L}_{G} \mathbf{x}}{\mathbf{x}^{\top} \mathbf{L}_{P} \mathbf{x}}=\max \frac{\left|\partial_{G}(Q)\right|}{\left|\partial_{P}(Q)\right|},
$$

where 1 is the all-one vector, the node set $Q$ is defined as $Q \stackrel{\text { def }}{=}$ $\{q \in V: x(q)=1\}$, and the boundary of $Q$ in $G$ is defined as $\partial_{G}(Q) \stackrel{\text { def }}{=}$ $\left\{(p, q) \in E_{G}: q \in Q, p \notin Q\right\}$, which obviously leads to $\mathbf{x}^{\top} \mathbf{L}_{G} \mathbf{x}=$ $\left|\partial_{G}(Q)\right|$, and $\mathbf{x}^{\top} \mathbf{L}_{P} \mathbf{x}=\left|\partial_{P}(Q)\right|$. Then (6) indicates that finding the dominant generalized eigenvector is approximately equivalent to the problem of finding $Q$ such that $\frac{\left|\partial_{G}(Q)\right|}{\left|\partial_{P}(Q)\right|}$ or the mismatch of boundary (cut) size between the original graph $G$ and subgraph $P$ is maximized. As a result, $\lambda_{\max }=\lambda_{1}$ is a good measure of the largest mismatch in boundary (cut) size between $G$ and $P$. Once $Q$ or $\partial_{P}(Q)$ is found via graph embedding using dominant generalized eigenvectors, we can sample the edges in $\partial_{G}(Q)$ and recover them to $P$ to dramatically decrease the maximum mismatch or $\lambda_{1}[10,11]$.

3.3.3 Subgraph Scaling via SGD Iterations. To aggressively limit the number of edges in the (reduced) subgraph while still achieving a high quality approximation of the original graph, we propose an efficient edge scaling scheme to mitigate the accuracy loss due to the missing nodes and edges. We propose to scale up edge weights in the (reduced) subgraph to further reduce the largest mismatch or $\lambda_{1}$ in (6).

Edge weight sensitivity for subgraph scaling. According to (5), the dominant eigenvalue perturbation $\delta \lambda_{1}$ with respect to edge weight perturbations will lead to:

$$
\begin{gathered}
-\frac{\delta \lambda_{1}}{\lambda_{1}}=\mathbf{u}_{1}^{\top} \delta \mathbf{L}_{P} \mathbf{u}_{1}=\sum_{(p, q) \in E_{P}} \delta w_{p q}\left(\mathbf{e}_{p q}^{\top} \mathbf{u}_{1}\right)^{2}, \\
=>\frac{\delta \lambda_{1}}{\delta w_{p q}}=-\lambda_{1}\left(\mathbf{e}_{p q}^{\top} \mathbf{u}_{1}\right)^{2} \approx-\lambda_{1}\left(\mathbf{e}_{p q}^{\top} \mathbf{h}_{t}\right)^{2} .
\end{gathered}
$$

where $\mathbf{h}_{t}$ is the approximate dominant eigenvector $\mathbf{u}_{1}$, which can be efficiently computed through a few steps of generalized power iterations [10]. With the (approximate) sensitivity expressed in (8), it is possible to find a proper weight scaling factor for each edge in $P$ such that $\lambda_{1}$ will be dramatically reduced. However, since both $\lambda_{1}$ and $\lambda_{n}$ will decrease monotonically when scaling up edge weights, it is likely that $\lambda_{n}$ will decrease at a faster rate than $\lambda_{1}$, which may even lead to a worse spectral approximation level. To address this issue, the latest nearly-linear time algorithms for estimating the extreme generalized eigenvalues are leveraged [11], which allows scaling up subgraph edges properly without degrading the spectral approximation in the subgraph.

Edge scaling via stochastic gradient descent (SGD) iterations. Based on the scalable methods [11] for estimating the extreme eigenvalues $\lambda_{1}$ and $\lambda_{n}$ of $\mathbf{L}_{P}^{+} \mathbf{L}_{G}$, as well as the weight sensitivity in (8), the following constrained nonlinear optimization framework can be leveraged for scaling up the subgraph $(P)$ edge 
weights $w_{P}$ to minimize the largest mismatch (generalized eigenvalue $\left.\lambda_{1}\right)$ [23]:

$$
\begin{aligned}
& \underset{w_{P}}{\operatorname{minimize}} \lambda_{1}\left(w_{P}\right) \\
& \text { subject to: } \\
& \text { 1) } \mathrm{L}_{G} \mathbf{u}_{i}=\lambda_{i} \mathbf{L}_{P} \mathbf{u}_{i}, i=1, \ldots, n \\
& \text { 2) } \lambda_{\max }=\lambda_{1} \geq \lambda_{2} \ldots \geq \lambda_{n}=\lambda_{\text {min }} \\
& \text { 3) } \lambda_{n} \geq \lambda_{n}^{(0)} \bar{\Delta}_{\lambda_{n}} .
\end{aligned}
$$

In (9), $\lambda_{n}^{(0)}$ denotes the smallest generalized eigenvalue before scaling and $\bar{\Delta}_{\lambda_{n}}$ denotes the maximum allowable reduction ratio of $\lambda_{n}$ with a value between 0 and 1 . To solve (9), a constrained SGD algorithm with momentum [20] has been developed in this work. Since the edge weights of the subgraph $\left(w_{P}\right)$ will be updated during each SGD iteration, we need to solve a new subgraph Laplacian matrix $\mathbf{L}_{P}$ for updating the approximate eigenvector $\mathbf{h}_{t}$ in (8), which can be efficiently achieved by leveraging recent graph-theoretic Laplacian solvers $[15,24]$. Since the subgraph topology remains unchanged during the SGD iterations, it is possible to exploit incremental update of graph Laplacian solvers to further improve efficiency. Details of the above algorithm can be found in [23].

\subsection{Phase (C): Effective-Resistance Preserving Post Scaling}

The last phase of our approach for spectral reduction of graphs is to globally scale up edge weights in the sparsified reduced graph to further improve the spectral approximation quality. Consider the following analysis for undirected graphs. Denote the descending eigenvalues and the corresponding unit-length, mutually-orthogonal eigenvectors of $\mathrm{L}_{G}$ by $\zeta_{1} \geq \cdots>\zeta_{n}=0$, and $\omega_{1}, \cdots, \omega_{n}$, respectively. Then the following spectral decompositions of $\mathrm{L}_{G}$ and $\mathrm{L}_{G}^{+}$ always hold:

$$
\mathbf{L}_{G}=\sum_{i=1}^{n-1} \zeta_{i} \omega_{i} \omega_{i}^{\top}, \mathbf{L}_{G}^{+}=\sum_{j=1}^{n-1} \frac{1}{\zeta_{j}} \omega_{j} \omega_{j}^{\top},
$$

which leads to the following effective resistance between nodes $p$ and $q$ in $G$ :

$$
R_{e}^{G}(p, q)=e_{p q}^{T} \mathbf{L}_{G}^{+} \mathbf{e}_{p q}=\sum_{i=1}^{n-1} \frac{1}{\zeta_{i}}\left(\mathbf{e}_{p q}^{T} \omega_{i}\right)^{2}
$$

(11) shows a close connection between the effective resistance metric and the first few Laplacian eigenvalues and eigenvectors. If we consider the graph as a resistor network with each conductance value corresponding to each edge weight, $R_{e}^{G}(p, q)$ can be regarded as the power dissipation of the resistor network when a unit current is flowing into node $p$ and out from node $q$. By replacing the current vector $\mathbf{e}_{p q}$ with a random vector $\mathrm{b}^{\perp} \in R^{n}$ orthogonal to the all-one vector, it can be shown that matching the energy dissipated in the reduced graph and the original graph will immediately lead to improved approximation of the first few Laplacian eigenvalues and eigenvectors associated with the reduced graph.

It should be noted that the above scheme requires solving the original and reduced Laplacians once for finding the scaling factor, which can be achieved in almost-linear time leveraging graphtheoretic Laplacian solvers [15, 24].

\subsection{Solution Refinement by Smoothing}

Denote the descending eigenvalues and the corresponding unitlength, mutually-orthogonal eigenvectors of $\mathbf{L}_{P}$ by $\tilde{\zeta}_{1} \geq \cdots>$ $\tilde{\zeta}_{n}=0$ and $\tilde{\omega}_{1}, \cdots, \tilde{\omega}_{n}$, respectively. We assume that the $k$ smallest eigenvalues and their eigenvectors of $\mathrm{L}_{G}$ have been precisely preserved in $\mathrm{L}_{P}$, while the remaining $n-k$ higher eigenvalues and eigenvectors are not. Then the following approximate spectral decomposition will hold based on (10):

$$
\mathbf{L}_{P} \approx \sum_{i=n-k+1}^{n} \zeta_{i} \omega_{\mathbf{i}} \omega_{i}^{\top}+\sum_{i=1}^{n-k} \tilde{\zeta}_{i} \tilde{\omega}_{\mathbf{i}} \tilde{\omega}_{\mathbf{i}}^{\top} .
$$

In the following, we show that using spectrally-sparsified graphs for solving sparse matrix problems will only result in solution errors that can be expressed with high eigenvectors, while the error analysis for spectrally-reduced graphs will be quite similar. Consider the following matrix solution problem: $\left(\mathbf{L}_{G}+\delta \mathbf{I}\right) \mathbf{x}=\mathbf{b}^{\perp}$, where $\delta$ is a very small positive real value for modeling boundary conditions, and $\mathbf{I} \in R^{n \times n}$ is an identity matrix. Let $\tilde{\mathbf{x}}$ denote the approximate solution obtained with $\mathbf{L}_{P}$, then the error vector $\mathbf{e}$ becomes:

$$
\begin{gathered}
\tilde{\mathbf{x}} \approx \sum_{i=1}^{n-k} \frac{\tilde{\omega}_{i} \tilde{\omega}_{i}^{\top} \mathbf{b}^{\perp}}{\tilde{\zeta}_{i}+\delta}+\sum_{i=n-k+1}^{n} \frac{\omega_{i} \omega_{i}^{\top} \mathbf{b}^{\perp}}{\zeta_{i}+\delta}, \\
=>\mathbf{e}=\mathbf{x}-\tilde{\mathbf{x}} \approx \sum_{i=1}^{n-k}\left(\frac{\omega_{i} \omega_{i}^{\top} \mathbf{b}^{\perp}}{\zeta_{i}+\delta}-\frac{\tilde{\omega}_{i} \tilde{\omega}_{i}^{\top} \mathbf{b}^{\perp}}{\tilde{\zeta}_{i}+\delta}\right),
\end{gathered}
$$

implying that when using the sparsified graph Laplacian for solving the SDD matrix, the solution error can be expressed as a linear combination of high eigenvectors corresponding to large Laplacian eigenvalues, which can be efficiently filtered out using "low-pass" graph signal filters [17]. To this end, modern iterative methods for solving large sparse matrices [16], such as the weighted Jacobi or Gauss-Seidel smoothing (relaxation) functions in multigrid algorithms [15], can be applied with the original Laplacian for filtering out such high-frequency errors introduced by spectrally-sparsified (reduced) graphs.

It is expected that spectrally-sparsified (reduced) graphs can be directly used with the proposed solution refinement scheme for approximately solving large SDD matrices or Laplacian eigenvalue problems. For example, nowadays on-chip power grid analysis tasks require to solve extremely large-scale SDD matrices corresponding to resistor networks consisting of billions of elements for computing power grid voltage and current distributions, which can be drastically accelerated by exploiting the proposed spectrally-sparsified (reduced) grids and solution refinement procedures; modern spectral graph partitioning and data clustering algorithms [14, 23] can be more scalable, when the eigendecomposition of Laplacian matrices is performed along with eigenvector solution refinement using spectrally-sparsified (reduced) graphs (data networks).

\subsection{Algorithm Complexity}

The algorithm complexity of Phase (A) for the spectrum-preserving node reduction procedure is $O\left(\left|E_{P}\right|\right)$ for dense graphs and $O\left(\left|E_{G}\right|\right)$ for sparse graphs, the complexity of Phase (B) for spectral graph sparsification and edge scaling by SGD iterations is $O\left(\left|E_{G}\right| \log (|V|)\right)$, while the complexity of Phase (C) for post scaling is $O\left(\left|E_{G}\right|\right.$ when the recent graph-theoretic AMG solvers are leveraged [15, 24] for solving the original Laplacian matrix $\mathrm{L}_{G}$. Therefore, the worse-case algorithm complexity of the proposed spectral graph reduction 


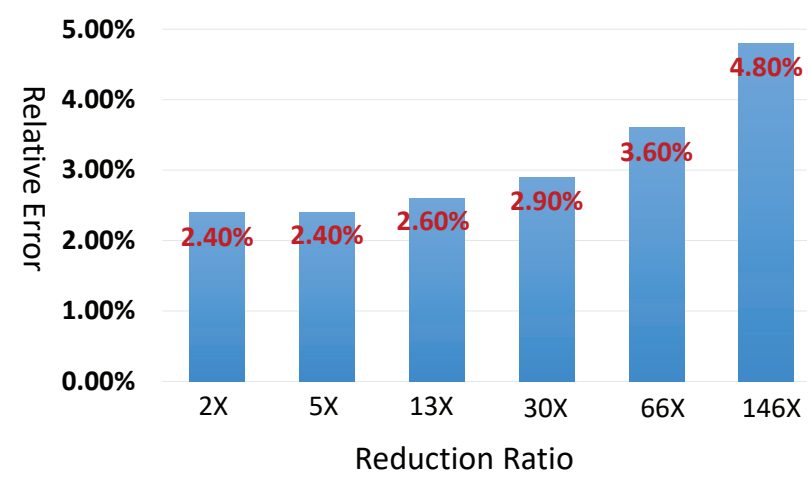

Figure 2: Average relative errors of effective resistance under different graph reduction ratios for the "fe_tooth" graph.

method is $O\left(\left|E_{G}\right| \log (|V|)\right)$. In addition, the solution refinement procedure obviously has a complexity of $O\left(\left|E_{G}\right|\right)$.

\section{EXPERIMENTAL RESULTS}

In this section, extensive experiments have been conducted to evaluate the proposed spectral graph reduction method with various types of graphs related to many different applications, such as finiteelement analysis problems ("fe_ocean", "fe_rotor", "parabolic_fem") [7], 3D integrated circuit (IC) thermal analysis ("3D_thermal"), power grid analysis ("Gmat_thu1"), image processing ("3D_laplacian"), star mixtures ("vsp_msc"), social networks ("coAuthorsDBLP", "coPapersDBLP" and "coPapersCiterseer") [2, 7], etc. All experiments have been conducted on a single $\mathrm{CPU}$ core of a computing platform running 64-bit RHEW 6.0 with $2.67 \mathrm{GHz} 12$-core $\mathrm{CPU}$ and $48 \mathrm{~GB}$ DRAM memory.

\subsection{Results of Spectral Graph Reduction}

Table 2 shows graph reduction results on different graphs using the proposed method, where effective resistances errors are reported. $\operatorname{Err}_{R}\left(\operatorname{Err}_{S}\right)$ denotes the average relative errors of effective resistances between graph $R(S)$ and graph $G ; T_{r}, T_{s}$ and $T_{t o t}$ denote the spectral reduction time, spectral graph sparsification with edge scaling time, and total reduction runtime, respectively. The relative errors of effective resistance values are computed by averaging the relative errors of the effective resistances computed for 100 randomly selected node pairs. The proposed solution refinement scheme with 20 weighted Jacobi relaxations has been applied for computing every effective resistance value in our results.

Compared to other test cases that correspond to sparse graphs, the graphs " $a p p u^{*}$ " and " $v s p \_m s c^{*}$ " have much higher densities and thus been processed as dense graphs. We want to emphasize that directly applying the prior algebraic-distance-based node aggregation scheme [5] for node reduction may not always produce acceptable results for dense graphs. For example, the node aggregation algorithm failed to generate the reduced graph for " $a p p u^{*}$ " due to its high graph density. On the other hand, there will be no issue when Phase (B) for spectral graph sparsification and scaling is applied before the node aggregation phase. For all test cases, it is observed that the effective resistances computed with the original graphs can be well approximated by using the spectrally reduced graphs $(\mathrm{R})$ and sparsified reduced graphs $(\mathrm{S})$. The average relative errors of 100 randomly computed effective resistances have been shown in Figure 2 with different spectral graph reduction ratios for the "fe_tooth" graph. We observe that the effective resistance accuracy will slight drop when higher reduction ratios are used.

\subsection{Results of Hypergraph Partitioning}

One approach to spectral hypergraph partitioning is to construct a graph from the hypergraph and then apply spectral graph partitioning to the generated graph [12]. Existing methods for constructing a graph from a hypergraph are based on clique or star expansions. In the case of clique expansion, each hyperedge is replaced by a complete subgraph for all vertices in that hyperedge. However, the size of the generated graph can be greatly increased. To achieve good efficiency, a multilevel Laplacian eigensolver has been developed to accelerate eigenvalue problems by leveraging spectrally-reduced graphs without loss of solution quality [23]: instead of directly computing the first $k$ eigenvectors of the original graph Laplacian $\mathbf{L}_{G}$, we first spectrally-reduce it into a much smaller Laplacian $\mathrm{L}_{S}$ for computing approximate eigenvectors that will be mapped onto a finer level using graph mapping operators $\left(\mathrm{H}_{\mathrm{R}}^{\mathrm{G}}\right)$. To further improve the approximation quality of these eigenvectors, an eigenvector refinement (smoothing) procedure similar to the solution refinement procedure has been applied. The eigenvector mapping and smoothing procedures are repeatedly applied until the finest level is reached. In the last, all the eigenvectors will be returned for spectral partitioning purpose (after being orthonormalized with the Gram-Schmidt process).

The performance of spectral hypergraph partitioning is evaluated on 18 hypergraphs from ISPD98 circuit partitioning benchmark suite [1], where only unit cell-areas are considered for the experiment. All hypergraphs are converted to corresponding graphs $G$ using clique representation, and the weight of each edge in the clique equals to $1 /(|e|-1)$, where $|e|$ is the number of vertices in the hyperedge. $k$-way spectral partitioning is performed on both graph $G$ and the spectrally-reduced graph $S$. Hyperedge cut and sum of external degrees (SOED) of all hyperedges that span multiple partitions are calculated to evaluate the partitioning quality. The overall performance of hypergraph partitioning with 8-, 16and 32-way partitions has been shown in Table 3, where "Cut" and "SD" denote the hyperedge cut and SOED metric, respectively; $T_{e}^{G}$ and $T_{e}^{S}$ denote the eigendecomposition time required by $G$ and $S$, respectively; $\frac{V}{V_{S}}$ and $\frac{E_{G}}{E_{S}}$ represent the node reduction ratio as well as the edge reduction ratio for spectral graph reduction. Comparing to the hypergraph partitioning using graph $G$, results show that partitioning with spectrally reduced graphs can produce comparable partitioning qualities with dramatically reduced cost.

\section{CONCLUSION}

We propose a scalable algorithmic framework for spectral reduction of large undirected graphs. The proposed method allows computing much smaller graphs while preserving the key spectrum of the original graph. Our spectral graph reduction framework is built upon a spectrum-preserving node aggregation scheme, a highly-scalable spectral graph sparsification and edge weight scaling scheme, as well as an effective-resistance preserving post scaling scheme. We show that the spectrally-reduced graphs produced by the proposed approach can robustly preserve the first few nontrivial eigenvalues and eigenvectors of the original graph Laplacian, effective resistance between nodes, etc, allowing for accelerating algorithms closely related to electronic design automation (EDA), numerical linear algebra, and social network applications. 
Table 2: Results of Effective-Resistance Preserving Spectral Graph Reduction.

\begin{tabular}{|c|c|c|c|c|c|c|c|c|c|c|c|}
\hline \multirow[b]{2}{*}{ Test Cases } & \multicolumn{2}{|c|}{ Original Graph $(G)$} & \multicolumn{4}{|c|}{ Reduced Graph w/o Sparsification $(R)$} & \multicolumn{4}{|c|}{ Reduced Graph w/ Sparsification $(S)$} & \multirow{2}{*}{$\frac{\text { Total time }}{T_{\text {tot }}(s)}$} \\
\hline & $|V|$ & $\left|E_{G}\right|$ & $\left|V_{R}\right|\left(\frac{|V|}{\left|V_{R}\right|}\right)$ & $\left|E_{R}\right|\left(\frac{\left|E_{G}\right|}{\left|E_{R}\right|}\right)$ & $\operatorname{Err}_{R}(\%)$ & $T_{r}(s)$ & $\left|V_{R}\right|\left(\frac{|V|}{\left|V_{R}\right|}\right)$ & $\left|E_{S}\right|\left(\frac{\left|E_{G}\right|}{\left|E_{S}\right|}\right)$ & $\operatorname{Err}_{S}(\%)$ & $T_{s}(s)$ & \\
\hline fe_rotor & $1.0 E 5$ & $6.6 E 5$ & $3.4 E 3(29 X)$ & $2.6 E 4(25 X)$ & $2.7 \%$ & $1.26 \mathrm{~s}$ & $3.4 E 3(29 X)$ & $6.9 E 3(95 X)$ & $2.4 \%$ & $3.54 \mathrm{~s}$ & $4.80 \mathrm{~s}$ \\
\hline fe_ocean & $1.4 E 5$ & $4.1 E 5$ & $6.1 E 3(24 X)$ & $3.2 E 4(13 X)$ & $7.7 \%$ & $1.02 \mathrm{~s}$ & $6.1 E 3(24 X)$ & $7.0 E 3(58 X)$ & $6.0 \%$ & $3.65 s$ & $4.67 s$ \\
\hline parabolic_fem & $5.3 E 5$ & $1.6 E 6$ & $1.1 E 4(46 X)$ & $3.4 E 4(47 X)$ & $7.9 \%$ & $3.94 s$ & $1.1 E 4(46 X)$ & $1.2 E 4(128 X)$ & $7.8 \%$ & $13.59 \mathrm{~s}$ & $17.53 s$ \\
\hline $2 D \_m e s h$ & $4.0 E 4$ & $8.0 E 4$ & $4.3 E 3(9 X)$ & $1.2 E 4(7 X)$ & $8.3 \%$ & $0.26 s$ & $4.3 E 3(9 X)$ & $4.7 E 3(17 X)$ & $7.2 \%$ & $1.59 \mathrm{~s}$ & $1.85 \mathrm{~s}$ \\
\hline 3D_thermal & $4.8 E 5$ & $1.4 E 6$ & $7.9 E 3(61 X)$ & $5.4 E 4(26 X)$ & $5.2 \%$ & $3.17 \mathrm{~s}$ & $7.9 E 3(61 X)$ & $8.7 E 3(163 X)$ & $4.4 \%$ & $11.18 \mathrm{~s}$ & $13.35 s$ \\
\hline $3 D \_l a p l a c i a n$ & $1.0 E 6$ & $3.0 E 6$ & $8.1 E 3(124 X)$ & $5.5 E 4(54 X)$ & $5.0 \%$ & $7.38 s$ & $8.1 E 3(124 X)$ & $8.9 E 3(334 X)$ & $4.3 \%$ & $18.66 \mathrm{~s}$ & $26.04 s$ \\
\hline Gmat_thu 1 & $5.0 E 6$ & $8.2 E 6$ & $9.7 E 4(51 X)$ & $2.9 E 5(29 X)$ & $9.2 \%$ & $26.52 s$ & $9.7 E 4(51 X)$ & $1.1 E 5(78 X)$ & $6.2 \%$ & $214.75 s$ & $241.27 s$ \\
\hline Gmat_airfoil & $4.2 E 3$ & $1.2 E 4$ & $8.3 E 2(5 X)$ & $2.3 E 3(5 X)$ & $7.8 \%$ & $0.07 s$ & $8.3 E 2(5 X)$ & $9.6 E 2(13 X)$ & $6.3 \%$ & $0.08 s$ & $0.15 s$ \\
\hline$a p p u^{*}$ & $1.4 E 4$ & $9.2 E 5$ & $1.3 E 2(107 X)$ & $7.0 E 3(131 X)$ & $0.1 \%$ & $9.53 s$ & $1.3 E 2(107 X)$ & $1.3 E 2(7,019 X)$ & $0.1 \%$ & $0.03 s$ & $9.56 s$ \\
\hline$v s p_{-} m s c^{*}$ & $2.2 E 4$ & $1.2 E 6$ & $2.2 E 2(100 X)$ & $4.4 E 3(280 X)$ & $5.6 \%$ & $11.16 \mathrm{~s}$ & $2.2 E 2(100 X)$ & $2.3 E 2(5,427 X)$ & $4.8 \%$ & $0.03 s$ & $11.19 \mathrm{~s}$ \\
\hline auto & $4.5 E 5$ & $3.3 E 6$ & $2.9 E 3(153 X)$ & $2.1 E 4(157 X)$ & $4.2 \%$ & $5.71 \mathrm{~s}$ & $2.9 E 3(153 X)$ & $3.1 E 3(1,079 X)$ & $4.2 \%$ & $11.71 \mathrm{~s}$ & $17.41 \mathrm{~s}$ \\
\hline coAuthorsDBLP & $3.0 E 5$ & $9.7 E 5$ & $1.3 E 3(233 X)$ & $5.5 E 4(18 X)$ & $3.3 \%$ & $2.36 \mathrm{~s}$ & $1.3 E 3(233 X)$ & $1.6 E 3(603 X)$ & $3.2 \%$ & $10.05 \mathrm{~s}$ & $12.41 \mathrm{~s}$ \\
\hline coPapersDBLP & $5.4 E 5$ & $1.5 E 7$ & $1.6 E 3(347 X)$ & $9.9 E 4(154 X)$ & $4.6 \%$ & $12.70 s$ & $1.6 E 3(347 X)$ & $1.6 E 3(9,336 X)$ & $4.6 \%$ & $21.25 s$ & $33.95 s$ \\
\hline coPapersCiteseer & $4.3 E 5$ & $1.6 E 7$ & $5.3 E 2(816 X)$ & $2.1 E 4(748 X)$ & $4.0 \%$ & $10.07 s$ & $5.3 E 2(816 X)$ & $5.6 E 2(28,843 X)$ & $3.6 \%$ & $16.69 \mathrm{~s}$ & $26.76 \mathrm{~s}$ \\
\hline
\end{tabular}

Table 3: Performance of Spectral Hypergraph Partitioning.

\begin{tabular}{|c|c|c|c|c|c|c|c|c|c|c|c|c|c|}
\hline \multirow{3}{*}{ ibm } & \multicolumn{6}{|c|}{ Partitioning w/ Graph $(G)$} & \multicolumn{7}{|c|}{ Partitioning w/ Reduced Graph $(S)$} \\
\hline & \multicolumn{2}{|c|}{ 8-way } & \multicolumn{2}{|c|}{ 16-way } & \multicolumn{2}{|c|}{ 32-way } & \multicolumn{2}{|c|}{ 8-way } & \multicolumn{2}{|c|}{ 16-way } & \multicolumn{2}{|c|}{ 32-way } & \multirow[b]{2}{*}{$\frac{V}{V_{S}}\left(\frac{E_{G}}{E_{S}}\right)$} \\
\hline & Cut (SD) & $T_{\boldsymbol{e}}^{G}$ & Cut (SD) & $T_{e}^{G}$ & Cut (SD) & $T_{e}^{G}$ & Cut (SD) & $T_{e}^{S}\left(\frac{T_{e}^{G}}{T_{e}^{S}}\right.$ & Cut (SD) & $T_{e}^{S}\left(\frac{T_{e}^{G}}{T_{e}^{S}}\right.$ & Cut (SD) & $T_{e}^{S}\left(\frac{T_{e}^{G}}{T_{e}^{S}}\right.$ & \\
\hline 01 & $800(1633)$ & $1.35 \mathrm{~s}$ & $943(1949)$ & $1.43 s$ & $1461(3057)$ & $1.94 \mathrm{~s}$ & $637(1295)$ & $0.08 s(17 X)$ & 914(1915) & $0.11 s(13 X)$ & $1471(3130)$ & $0.18 s(11 X)$ & $13 X(63 X)$ \\
\hline 02 & $1096(2302)$ & $4.44 s$ & $2039(4244)$ & $5.70 \mathrm{~s}$ & $3163(6695)$ & $8.27 \mathrm{~s}$ & $1034(2204)$ & $0.08 s(56 X)$ & $2173(4654)$ & $0.17 s(34 X)$ & $3415(7426)$ & $0.25 s(33 X)$ & $12 X(97 X)$ \\
\hline 03 & $1532(3089)$ & $6.29 s$ & $2710(5542)$ & $7.26 \mathrm{~s}$ & $4419(9166)$ & $11.53 \mathrm{~s}$ & $1549(3124)$ & $0.18 s(35 X)$ & $2729(5627)$ & $0.23 s(32 X)$ & $4483(9520)$ & $0.33 s(35 X)$ & $5 X(35 X)$ \\
\hline 04 & $1995(4108)$ & $5.40 \mathrm{~s}$ & $3127(6614)$ & $7.43 \mathrm{~s}$ & $4727(9942)$ & $10.58 \mathrm{~s}$ & $2012(4093)$ & $0.11 s(49 X)$ & $3256(6840)$ & $0.18 s(41 X)$ & $4869(10387)$ & $0.27 s(39 X)$ & $5 X(40 X)$ \\
\hline 05 & $3100(6263)$ & $25.69 s$ & $4472(9208)$ & $53.86 s$ & $5289(10865)$ & $63.12 s$ & $3144(6417)$ & $1.17 s(22 X)$ & $4680(9946)$ & $1.65 s(33 X)$ & $5606(11237)$ & $2.30 s(27 X)$ & $5 X(21 X)$ \\
\hline \begin{tabular}{|l|}
06 \\
\end{tabular} & $2390(4960)$ & $12.10 \mathrm{~s}$ & $3367(7193)$ & 15.90 & $4507(9611)$ & $20.88 s$ & 1742(3587) & $0.17 s(71 X)$ & $3460(7264)$ & $0.35 s(45 X)$ & $4545(9851)$ & $0.51 s(41 X)$ & $12 X(51 X)$ \\
\hline \begin{tabular}{|l|}
07 \\
\end{tabular} & $1724(3508)$ & $13.71 s$ & $3785(7666)$ & $18.72 s$ & 6713(13968) & $23.36 s$ & $1643(3404)$ & $0.14 s(98 X)$ & $3925(8069)$ & $0.23 s(81 X)$ & 6373(13495) & $0.29 s(81 X)$ & $13 X(69 X)$ \\
\hline \begin{tabular}{|l|}
08 \\
\end{tabular} & $3343(6815)$ & $32.35 s$ & $4750(9848)$ & $33.69 s$ & 6318(13117) & $47.05 s$ & 3291 (6747) & $0.51 s(63 X)$ & 4916(10126) & $0.75 s(45 X)$ & 6041(12825) & $1.02 s(46 X)$ & $11 X(53 X)$ \\
\hline \begin{tabular}{|l|}
09 \\
\end{tabular} & $2257(4569)$ & $11.56 \mathrm{~s}$ & $4508(9234)$ & $13.35 s$ & $5370(11217)$ & \begin{tabular}{|l|}
$21.47 s$ \\
\end{tabular} & $2164(4379)$ & $0.25 s(46 X)$ & $3241(6740)$ & $0.42 s(32 X)$ & $4467(9163)$ & $0.71 s(30 X)$ & $13 X(38 X)$ \\
\hline \begin{tabular}{|l|}
10 \\
\end{tabular} & $3043(6126)$ & 32.33 & $4468(9002)$ & $44.01 \mathrm{~s}$ & 5954(12291) & \begin{tabular}{|l|}
$58.78 s$ \\
\end{tabular} & $3104(6275)$ & $0.20 s(162 X)$ & $4847(9794)$ & $0.31 s(142 X)$ & 6324(13014) & $0.42 s(140 X)$ & $12 X(79 X)$ \\
\hline \begin{tabular}{|l|}
11 \\
\end{tabular} & $3305(6674)$ & $18.38 s$ & $4629(9514)$ & $22.13 s$ & $7169(14875)$ & $31.87 s$ & $3220(6504)$ & $0.40 s(46 X)$ & 4972(10070) & $0.58 s(38 X)$ & $7396(15671)$ & $0.78 s(41 X)$ & $12 X(39 X)$ \\
\hline \begin{tabular}{|l|}
12 \\
\end{tabular} & $2575(5162)$ & $69.74 s$ & $4538(9201)$ & $99.08 s$ & $7052(14446)$ & $103.11 s$ & $2323(4658)$ & $0.42 s(166 X)$ & $4622(9395)$ & $0.56 s(1774 X)$ & $6890(14140)$ & $1.15 s(90 X)$ & $5 X(41 X)$ \\
\hline \begin{tabular}{|c|}
13 \\
\end{tabular} & $1438(2957)$ & $20.87 s$ & $3742(7841)$ & $25.69 s$ & $6470(13448)$ & $36.35 s$ & $1561(3156)$ & $0.18 s(116 X)$ & $3849(7912)$ & $0.25 s(103 X)$ & $6934(14759)$ & $0.40 s(91 X)$ & $11 X(76 X)$ \\
\hline 14 & $5140(10376)$ & $83.64 s$ & $8504(17341)$ & $96.19 s$ & $12905(26541)$ & $161.56 s$ & $3726(7475)$ & $0.24 s(349 X)$ & $7975(16115)$ & $0.39 s(247 X)$ & $11779(24282)$ & $0.52 s(311 X)$ & $14 X(83 X)$ \\
\hline 15 & $3696(7435)$ & $121.86 s$ & \begin{tabular}{|l|}
$8416(17183)$ \\
\end{tabular} & $144.45 s$ & $14598(30426)$ & $207.70 s$ & $3727(7506)$ & $0.33 s(369 X)$ & \begin{tabular}{|l|}
$7431(14926)$ \\
\end{tabular} & $0.47 s(307 X)$ & $12738(26387)$ & $0.85 s(244 X)$ & $11 X(99 X)$ \\
\hline \begin{tabular}{|l|}
16 \\
\end{tabular} & 6301(12751) & $172.66 s$ & \begin{tabular}{|l|}
$9250(18810)$ \\
\end{tabular} & $255.15 s$ & $14439(29858)$ & $363.61 s$ & $6279(12646)$ & $0.39 s(443 X)$ & $10090(20543)$ & $0.66 s(387 X)$ & $14894(30695)$ & $0.91 s(400 X)$ & $12 X(101 X)$ \\
\hline 17 & $7710(15710)$ & $551.31 s$ & $10724(21915)$ & $643.25 s$ & $14367(29459)$ & $763.69 s$ & $7874(15970)$ & $0.92 s(599 X)$ & $10141(20716)$ & $1.17 s(550 X)$ & $16172(33366)$ & $1.74 s(439 X)$ & $11 X(95 X)$ \\
\hline 18 & $3902(7858)$ & $184.27 s$ & $6181(12447)$ & $208.02 s$ & $8313(16894)$ & $211.84 s$ & $3252(6529)$ & $0.35 s(526 X)$ & $5947(12012)$ & $0.38 s(547 X)$ & $8128(16610)$ & $0.55 s(385 X)$ & $11 X(107 X)$ \\
\hline
\end{tabular}

\section{ACKNOWLEDGMENTS}

This work is supported in part by a research gift from KeySight Technologies, as well as the grants CCF-1350206 (CAREER) and CCF-1618364 (SHF) from the National Science Foundation.

\section{REFERENCES}

[1] C. J. Alpert. The ispd98 circuit benchmark suite. In Proceedings of the 1998 international symposium on Physical design, pages 80-85. ACM, 1998.

[2] D. A. Bader, H. Meyerhenke, P. Sanders, and D. Wagner. Graph partitioning and graph clustering. In 10th DIMACS Implementation Challenge Workshop, 2012.

[3] J. Batson, D. Spielman, and N. Srivastava. Twice-Ramanujan Sparsifiers. SIAM Journal on Computing, 41(6):1704-1721, 2012.

[4] W. L. Briggs, S. F. McCormick, et al. A multigrid tutorial, volume 72. Siam, 2000.

[5] J. Chen and I. Safro. Algebraic distance on graphs. SIAM Journal on Scientific Computing, 33(6):3468-3490, 2011.

[6] P. Christiano, J. Kelner, A. Madry, D. Spielman, and S. Teng. Electrical flows, laplacian systems, and faster approximation of maximum flow in undirected graphs. In Proc. ACM STOC, pages 273-282, 2011.

[7] T. Davis and Y. Hu. The university of florida sparse matrix collection. ACM Trans. on Math. Soft. (TOMS), 38(1):1, 2011

[8] M. Defferrard, X. Bresson, and P. Vandergheynst. Convolutional neural networks on graphs with fast localized spectral filtering. In Advances in Neural Information Processing Systems, pages 3844-3852, 2016.

[9] M. Elkin, Y. Emek, D. A. Spielman, and N. Srivastava. Lower-stretch spanning trees. SIAM fournal on Computing, 38(2):608-628, 2008.

[10] Z. Feng. Spectral graph sparsification in nearly-linear time leveraging efficient spectral perturbation analysis. In Design Automation Conference (DAC), 2016 53nd ACM/EDAC/IEEE, pages 1-6. IEEE, 2016.

[11] Z. Feng. Similarity-aware spectral sparsification by edge filtering. In Design Automation Conference (DAC), 2018 55nd ACM/EDAC/IEEE. IEEE, 2018.
[12] G. Karypis, R. Aggarwal, V. Kumar, and S. Shekhar. Multilevel hypergraph partitioning: applications in vlsi domain. IEEE Transactions on Very Large Scale Integration (VLSI) Systems, 7(1):69-79, 1999.

[13] Y. Koren. On spectral graph drawing. In International Computing and Combinatorics Conference, pages 496-508. Springer, 2003.

[14] J. R. Lee, S. O. Gharan, and L. Trevisan. Multiway spectral partitioning and higher-order cheeger inequalities. Fournal of the ACM (7ACM), 61(6):37, 2014.

[15] O. Livne and A. Brandt. Lean algebraic multigrid (LAMG): Fast graph Laplacian linear solver. SIAM Journal on Scientific Computing, 34(4):B499-B522, 2012.

[16] Y. Saad. Iterative methods for sparse linear systems, volume 82. siam, 2003.

[17] D. I. Shuman, S. K. Narang, P. Frossard, A. Ortega, and P. Vandergheynst. The emerging field of signal processing on graphs: Extending high-dimensional data analysis to networks and other irregular domains. IEEE Signal Processing Magazine, 30(3):83-98, 2013.

[18] D. Spielman and S. Teng. Nearly linear time algorithms for preconditioning and solving symmetric, diagonally dominant linear systems. SIAM fournal on Matrix Analysis and Applications, 35(3):835-885, 2014.

[19] D. A. Spielman and N. Srivastava. Graph sparsification by effective resistances. SIAM Journal on Computing, 40(6):1913-1926, 2011.

[20] I. Sutskever, J. Martens, G. Dahl, and G. Hinton. On the importance of initialization and momentum in deep learning. In International conference on machine learning, pages 1139-1147, 2013.

[21] S.-H. Teng. Scalable algorithms for data and network analysis. Foundations and Trends ${ }^{\circledR}$ in Theoretical Computer Science, 12(1-2):1-274, 2016.

[22] Z. Zhao and Z. Feng. A spectral graph sparsification approach to scalable vectorless power grid integrity verification. In Proceedings of the 54th Annual Design Automation Conference 2017, page 68. ACM, 2017.

[23] Z. Zhao, Y. Wang, and Z. Feng. Nearly-linear time spectral graph reduction for scalable graph partitioning and data visualization. arXiv preprint arXiv:1812.08942.

[24] Z. Zhao, Y. Wang, and Z. Feng. SAMG: Sparsified graph theoretic algebraic multigrid for solving large symmetric diagonally dominant (SDD) matrices. In Proceedings of ACM/IEEE International Conference on Computer-Aided Design, pages 601-606, 2017. 